\title{
Napabucasin Reduces Cancer Stem Cell Characteristics in Hepatocellular Carcinoma
}

\author{
Ya Li, Qiuju Han, Huajun Zhao, Quanjuan Guo and Jian Zhang* \\ Institute of Immunopharmaceutical Sciences, School of Pharmaceutical Sciences, Shandong University, Jinan, China
}

\section{OPEN ACCESS}

Edited by: Patricia Sancho,

Universidad de Zaragoza, Spain

Reviewed by:
Maksim Sinyuk,
Lerner Research Institute,
United States
Silvia Peppicelli,
University of Florence, Italy
${ }^{*}$ Correspondence:
Jian Zhang
zhangj65@sdu.edu.cn

Specialty section:

This article was submitted to Pharmacology of Anti-Cancer Drugs,

a section of the journal

Frontiers in Pharmacology

Received: 21 August 2020

Accepted: 29 October 2020

Published: 03 December 2020

Citation:

LiY, Han Q, Zhao H, Guo Q and Zhang $J$ (2020) Napabucasin Reduces Cancer Stem Cell Characteristics in Hepatocellular Carcinoma. Front. Pharmacol. 11:597520. doi: 10.3389/fphar.2020.597520
Hepatocellular carcinoma (HCC) is the most common type of primary liver cancer. Cancer stem cells (CSCs) are a rare population with self-renewal and multipotent differentiation capacity, and reside among the more differentiated cancer cells. CSCs are associated with tumor recurrence, drug resistance and poor prognosis. The aim of this study was to determine the efficacy of napabucasin against $\mathrm{HCC}$ and elucidate the underlying molecular mechanisms. Napabucasin significantly decreased the viability of HCC cells in vitro by inducing apoptosis and cell cycle arrest. In addition, it suppressed CSC-related gene expression and spheroid formation in vitro, indicating depletion of CSCs. The antineoplastic effects of napabucasin was also evident in homograft tumor-bearing mouse models. Our findings provide the scientific basis of conducting clinical trials on napabucasin as a new therapeutic agent against HCC.

Keywords: napabucasin, stat3, hepatocellular carcinoma, cancer stem cells, stemness, hepatitis B virus

\section{INTRODUCTION}

Hepatocellular carcinoma (HCC) is the most common type of primary liver cancer, and chronic liver diseases and cirrhosis are its underlying pathologies. The annual mortality rate of liver cancer is 700,000 worldwide, and the number of newly diagnosed HCC is estimated to increase further in the coming decades (Balogh et al., 2016; Rawla et al., 2018; Asrani et al., 2019). Since it is asymptomatic in the early stages, HCC is usually diagnosed in its advanced stage, which precludes the possibility of surgical resection (Daher et al., 2018). At present, sorafenib is the only chemotherapeutic agent approved for treating advanced HCC, although it provides a survival benefit of only a few months (Geschwind and Chapiro, 2016; Samonakis and Kouroumalis, 2017; Daher et al., 2018). Therefore, early diagnosis and treatment of HCC is currently a major research focus.

Cancer stem cells (CSCs) are a rare subpopulation of tumor cells that have the capacity to selfrenew and differentiate into multiple lineages. They exist along with the larger differentiated cancer cells, and are associated with tumor recurrence and poor prognosis (Jiang et al., 2012; Bao et al., 2013). The self-renewal of CSCs is regulated by transcription factors like Nanog, SOX2, Oct 4 and Klf4 (Frank et al., 2010). CSCs are also responsible for tumor radio-resistance, chemoresistance and invasion (Phi et al., 2018). Therefore, preventing liver CSCs formation is a viable therapeutic strategy against HCC.

Signal transducer and activator of transcription 3 (STAT3) is a latent cytoplasmic transcription factor that translocates to the nucleus following tyrosine phosphorylation at position 705 and dimerization (Karras et al., 1997). STAT3 activation is tightly controlled in healthy cells, and is constitutively activated during tumorigenesis, where in it upregulates genes involved in tumor cell proliferation, invasion, migration, and angiogenesis (Kamran et al., 2013). STAT3 is overexpressed in 
approximately $60 \%$ of the HCC tissues, and is associated with poor prognosis (Kim et al., 2004). Consistent with this, blocking the STAT3 signaling pathway in human HCC cells using a decoy oligonucleotide induced apoptosis (Sun et al., 2008). Furthermore, STAT3 controls the expression of the CSC transcription factors like October 4 and Nanog (Yin et al., 2015). Won et al. reported that interleukin-6 (IL-6)-mediated STAT3 signaling and hypoxia upregulated CD133 in HCC cells and promoted tumor progression (Won et al., 2015). IL-6/ STAT3-dependent Oct4 expression has also been observed in HCC cell lines and tumor homografts (Lai et al., 2018). Therefore, STAT3 is a promising therapeutic target in HCC.

Napabucasin is a neoteric small molecule that targets the STAT3 pathway, and has shown encouraging results in phase III clinical trials on metastatic colorectal carcinoma, pancreatic cancer, gastric cancer and non-small cell lung cancer (Sonbol et al., 2019). In addition, napabucasin prevented relapse and metastasis of human tumor xenografts by inhibiting CSCs ( $\mathrm{Li}$ et al., 2015; Beyreis et al., 2019). However, the efficacy of napabucasin in HCC and the underlying molecular mechanisms have not been elucidated. Here, we found that napabucasin suppressed HCC cell growth in vitro and in vivo, and was highly toxic to HCC-derived CSCs. Our findings indicate that napabucasin is a promising agent for treating HCC.

\section{MATERIALS AND METHODS}

\subsection{Cell Lines and Culture}

The human HCC cell lines Huh7, HepG2 and the murine Hepa16 cells were purchased from the Cell Bank of Type Culture Collection of the Chinese Academy of Sciences (Shanghai, China), human HCC cell line HepG2.2.15 was kindly provided by professor Chun-Hong Ma (School of Basic Medical Science, Shandong University, China). All of the cells were cultured in DMEM or RPMI-1640 medium supplemented with $10 \%$ fetal bovine serum (FBS; Biological Industries, CT, USA) and $1 \%$ penicillin and streptomycin (Solarbio, Beijing, China) at $37^{\circ} \mathrm{C}$ under $5 \% \mathrm{CO}_{2}$. The HepG2.2.15 cells were selectively cultured with $200 \mathrm{mg} / \mathrm{ml}$ antibiotic G418 (Sigma-Aldrich, St. Louis, MS, USA).

\subsection{Drugs}

The STAT3 inhibitor napabucasin, cryptotanshinone and oxaliplatin were purchased from Selleck (Selleckchem, Houston, TX, USA). Napabucasin and cryptotanshinone were dissolved in dimethyl sulfoxide (DMSO) (Solarbio) at the final concentration of $50 \mathrm{mM}$ as stock solution and stored at $-20^{\circ} \mathrm{C}$. Oxaliplatin was dissolved in saline at $10 \mathrm{mM}$ for the stock solution.

\subsection{Cell Viability Assay}

HCC cells were seeded in a 96-well plate at the density of $1 \times 10^{4}$ cells/200 $\mu \mathrm{L} /$ well in DMEM or RPMI-1640 medium containing different concentrations of napabucasin, cryptotanshinone or oxaliplatin. After culturing the cells for 24,48 or $72 \mathrm{~h}$, cell counting kit-8 (CCK8, Beyotime Biotechnology, Shanghai,
China) was added to each well and the cells were incubated for another $1 \mathrm{~h}$. Absorbance was measured at $450 \mathrm{~nm}$ using a Synergy $^{\mathrm{TM}}$ two Multi-Mode microplate spectrophotometer (BioTek, Winooski, VT, USA).

\subsection{Apoptosis Assay}

HCC cells were seeded in a 6-well plate at the density of $2 \times 10^{5}$ cells per well, cultured overnight, and incubated with napabucasin for an additional 4 or $12 \mathrm{~h}$. The cells were harvested, washed with phosphate buffered saline (PBS), and stained using the Annexin V-FITC Apoptosis Detection Kit (Sungene biotech, Tianjin, China) according to the manufacturer's instructions. The stained cells were acquired by FACSCalibur flow cytometer (BD Biosciences, Franklin Lakes, NJ, USA), and the percentages of apoptotic cells were analyzed using the FlowJo software (Tree Star, San Carlos, CA, USA).

\subsection{Cell Cycle Analysis}

$\mathrm{HCC}$ cells were harvested and fixed with $70 \%$ ethanol in $\mathrm{ddH}_{2} \mathrm{O}$ at room temperature for $2 \mathrm{~h}$, washed with PBS, and incubated with propidium iodide (PI) solution (Sungene biotech, Tianjin, China) for $30 \mathrm{~min}$. The cells were sorted by FACSCalibur flow cytometer (BD Biosciences), and the cell cycle distribution was analyzed using ModFit LT 5.0.

\subsection{Hoechst Staining}

Suitably-treated HCC cells were fixed with $4 \%$ paraformaldehyde (Sinopharm Chemical Reagent limited corporation, Shanghai, China) and stained with $1 \mu \mathrm{g} / \mathrm{ml}$ Hoechst 33342 (Beyotime Biotechnology). The cells were observed by fluorescence microscopy (Olympus Corporation, Tokyo, Japan).

\subsection{Colony Formation Assay}

HCC cells were seeded in a 6-well plate at the density of 500 cells/ well, and incubated with DMSO or varying concentrations of napabucasin for $8 \mathrm{~h}$, followed by a 7-days culture. The cells were fixed with $4 \%$ paraformaldehyde (Sinopharm Chemical Reagent limited corporation) and stained with $1 \%$ crystal violet solution (Solarbio) for $10 \mathrm{~min}$, and the colonies (>100 cells) were counted under a microscope (Olympus Corporation).

\subsection{Spheroid Culture}

For testing self-renewal capacity, a total of 100 single HCC cells were seeded in each well of ultra-low attachment 96-well plate (Corning Incorporated, Corning, NY, USA) in complete suspension culture medium, DMEM/F12 (Invitrogen, Carlsbad, CA, USA) containing 2\% B27 (Invitrogen), 1\% N2 (Invitrogen), $20 \mathrm{ng} / \mathrm{ml}$ epidermal growth factor (EGF; Pep-Tech Corporation, Burlington, MA, USA), and $20 \mathrm{ng} / \mathrm{ml}$ basic fibroblast growth factor (bFGF; PepTech Corporation) with DMSO or various concentrations of napabucasin. The multicellular spheroids larger than $80 \mu \mathrm{m}$ were counted after 7 days.

For extreme limiting dilution analysis (ELDA), the HCC multicellular spheroids were dissociated to single cells and seeded at different densities (1, 10, 50 and 100/well) in complete suspension culture medium with DMSO or $1 \mu \mathrm{M}$ napabucasin in ultra-low attachment 96-well plates. There 

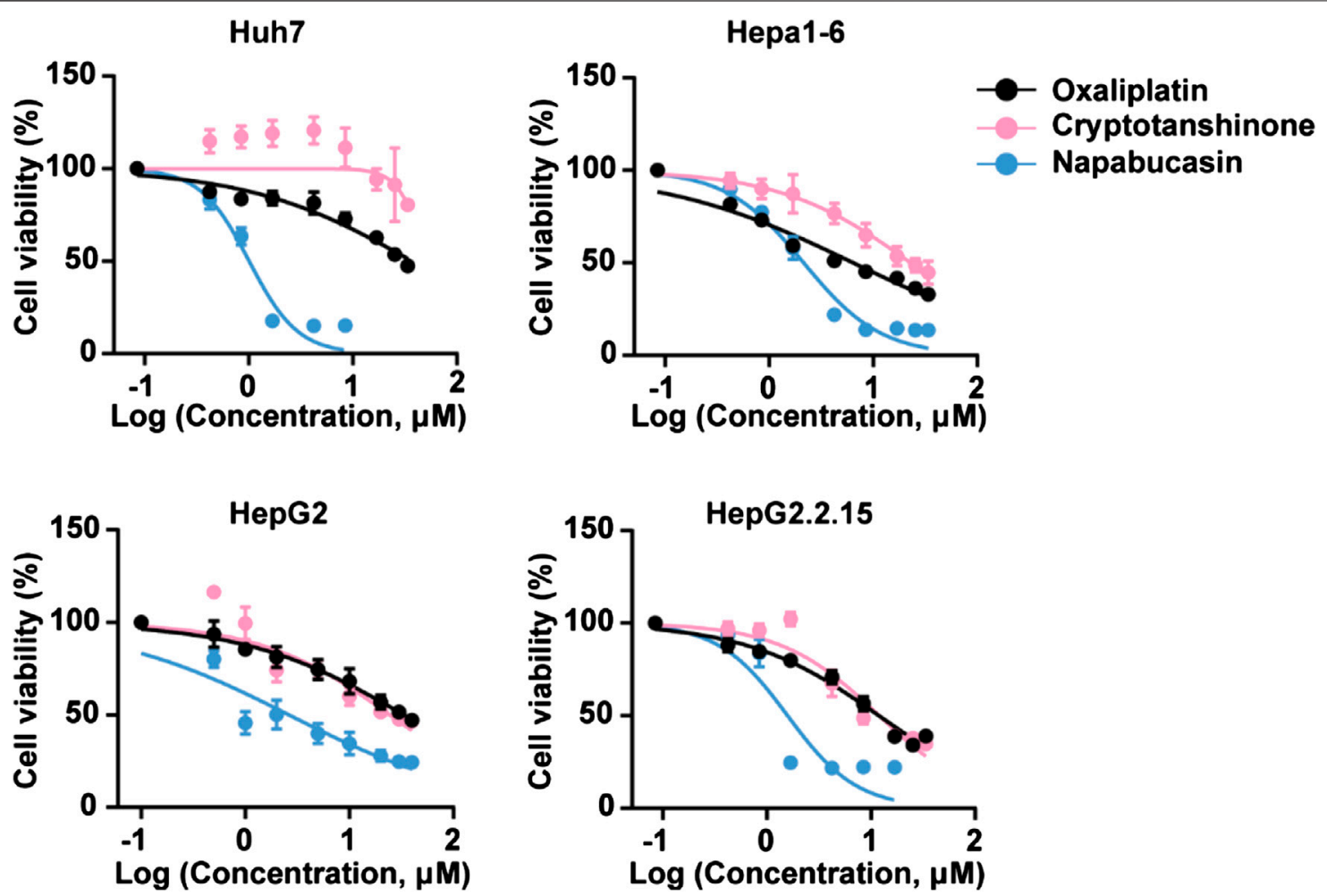

FIGURE 1 | Napabucasin decreases the viability of HCC cells in vitro. Viability of HCC cells incubated with DMSO or indicated concentrations of oxaliplatin, cryptotanshinone and napabucasin for $48 \mathrm{~h}$. Data are representative of three independent experiments.

were nine wells per cell density and cultured for 7 days. The numbers of wells with at least one multicellular spheroids (diameter $>80 \mu \mathrm{m}$ ) were counted in a blinded manner. Frequencies of sphere-initiating cells were calculated by ELDA online program (http://bioinf.wehi.edu.au/software/elda/) (Hu and Smyth, 2009).

To evaluate the effect of napabucasin on HCC multicellular spheroids, a total of 500 single HCC cells were seeded in each well of ultra-low attachment 6-well plate (Corning Incorporated) in complete suspension culture medium. The multicellular spheroids larger than $80 \mu \mathrm{m}$ were harvested for analysis 7 days after growth. For subsequent treatment with napabucasin, HCC multicellular spheroids (10 per well) were seeded into ultra-low attachment 96-well plate (Corning Incorporated) in complete suspension culture medium with DMSO or various concentrations of napabucasin. The images were captured with the JuLI ${ }^{\mathrm{TM}}$ Stage Real-Time Cell History Recorder (NanoEnTek, Seoul, South Korea).

\subsection{Western Blotting}

Cellular proteins were extracted using RIPA buffer (Beyotime Biotechnology), and equal amounts per sample were separated by SDS-PAGE in an $8 \%$ gel. The protein bands were transferred to a polyvinylidene-fluoride (PVDF) membrane (Millipore, Burlington, MA, USA) that was blocked with 5\% fat-free milk in PBS, and then incubated overnight with anti-p-STAT3 (Tyr705) (Cell Signaling Technology, Danvers, MA, USA),
anti-STAT3 (Cell Signaling Technology) and anti-GAPDH (Beyotime Biotechnology) antibodies (diluted 1:1000). The blots were further incubated with HRP-conjugated goat antirabbit or mouse IgG secondary antibodies (Beyotime Biotechnology) and developed with enhanced chemiluminescence reagent (Millipore, Billerica, MA, USA). The density of the bands was calculated using the Image-Lab software (Version 3.0, Bio-Rad).

\subsection{Quantitative Real-Time PCR (qRT-PCR)} Suitably-treated HCC cells were washed with $1 \times$ PBS 3 times before collected. Total RNA from HCC cells was extracted using TRIzol reagent (Invitrogen) and reverse transcribed to cDNAs

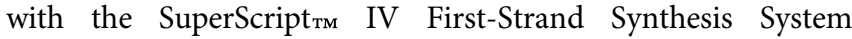
(Invitrogen, Carlsbad, CA, USA). Quantitative RT-PCR was performed using SYBR Green I Master (Roche, Basel, Switzerland) on a LightCycler ${ }^{\circledR} 480$ System according to the manufacturer's instructions. The relative mRNA levels were calculated using the $2^{-\Delta \Delta C t}$ method. Primer sequences are shown in Supplementary Table 1.

\subsection{Mice and Tumor Models}

C57BL/6J male mice (6-week-old) were purchased from Huafukang Laboratories (Beijing, China), and all animal experiments were performed according to the recommendations of the Ethical Committee of Shandong University. The mice were each subcutaneously injected in 
TABLE 1 | IC $\mathrm{C}_{50}$ of napabucasin, oxaliplatin and cryptotanshinone in $\mathrm{HCC}$ cell lines.

\begin{tabular}{lcccc}
\hline IC $_{\mathbf{5 0}}(\boldsymbol{\mu} \mathbf{M})$ & Huh7 & HepG2 & HepG2.2.15 & Hepa1-6 \\
\hline Oxaliplatin & $44.09 \pm 0.79$ & $33.53 \pm 0.91$ & $14.09 \pm 0.34$ & $7.56 \pm 2.7$ \\
Cryptotanshinone & $61.31 \pm 2.3$ & $24.49 \pm 0.97$ & $14.19 \pm 1.59$ & $26.95 \pm 1.17$ \\
Napabucasin & $1.2 \pm 0.13$ & $2.64 \pm 0.03$ & $1.88 \pm 0.17$ & $2.53 \pm 0.23$
\end{tabular}

their left axilla with $5 \times 10^{6}$ Hepa1-6 cells, and the tumors were measured every 2 days. The tumor volume $\left(\mathrm{mm}^{3}\right)$ was calculated as length $\times$ width $^{2} / 2$. The tumor-bearing mice were intraperitoneally injected with $20 \mathrm{mg} / \mathrm{kg}$ napabucasin every 2 days in a 1:8:1:10 napabucasin:poly (ethylene glycol)-300: Tween 80:saline mix, or equal volume of the vehicle. The mice were euthanized after eight injections, and the tumors were harvested for further analysis.

\subsection{Isolation of Mouse Tumor Cells}

HCC tumor tissues were harvested, minced, and digested with $0.1 \mathrm{mg} / \mathrm{ml}$ DNAse (Roche), $1 \mathrm{mg} / \mathrm{ml}$ type IV collagenase (Invitrogen) and $0.5 \%$ hyaluronic acid (Solarbio) for $1 \mathrm{~h}$ at $37^{\circ} \mathrm{C}$. The homogenates were passed through 200-mesh stainless steel strainers to obtain single cell suspension. The tumor cells were pelleted with $700 \mathrm{rpm}$ for $1 \mathrm{~min}$ at the bottom of the centrifuge tube. For qRT-PCR analysis, total RNAs were extracted with TRIzol. For flow cytometry analysis, cells were fixed with $4 \%$ paraformaldehyde and $0.5 \%$
TritonX-100 (Sigma), blocked with $0.1 \%$ rat serum in PBS and stained with fluorescence-conjugated anti-Ki67 antibody (Clone 16A8, Biolegend, San Diego, CA, USA) for $40 \mathrm{~min}$ at $4^{\circ} \mathrm{C}$. The stained cells were acquired using a FACSCalibur flow cytometer (BD Biosciences) and analyzed by FlowJo software (Tree Star).

\subsection{Histology and Immunohistochemistry}

Tumor homografts were excised after treatment, fixed with $4 \%$ paraformaldehyde, embedded in paraffin, and cut into $4-\mu \mathrm{m}$ thick sections. Hematoxylin and eosin (H\&E) staining was performed as per standard protocols, and the necrotic areas were observed under a light microscope (Olympus Corporation). For TUNEL staining, the tissue slices were deparaffinized with xylene, successively rehydrated in $100 \%, 90 \%, 80 \%$ and $70 \%$ ethanol, and incubated with $20 \mu \mathrm{g} / \mathrm{ml}$ Proteinase $\mathrm{K}$ without DNase for $30 \mathrm{~min}$. After washing with PBS, the slices were incubated with the TUNEL working solution for $1 \mathrm{~h}$ at room temperature, and observed under a fluorescence microscope (Olympus Corporation).

\subsection{STAT3 Interference}

The sequence 5'-TGCTGACCAACAATCCCAA-3' (residues 1663-1681 in the human STAT3 cDNA sequence, GenBank accession no. NM_003150) as a target to construct shRNA for stable knockdown of STAT3 was cloned into pLKO.1 plasmid. The recombinant lentiviruses expressing the STAT3 interference fragment were produced in $293 \mathrm{~T}$ cells and used to infect human

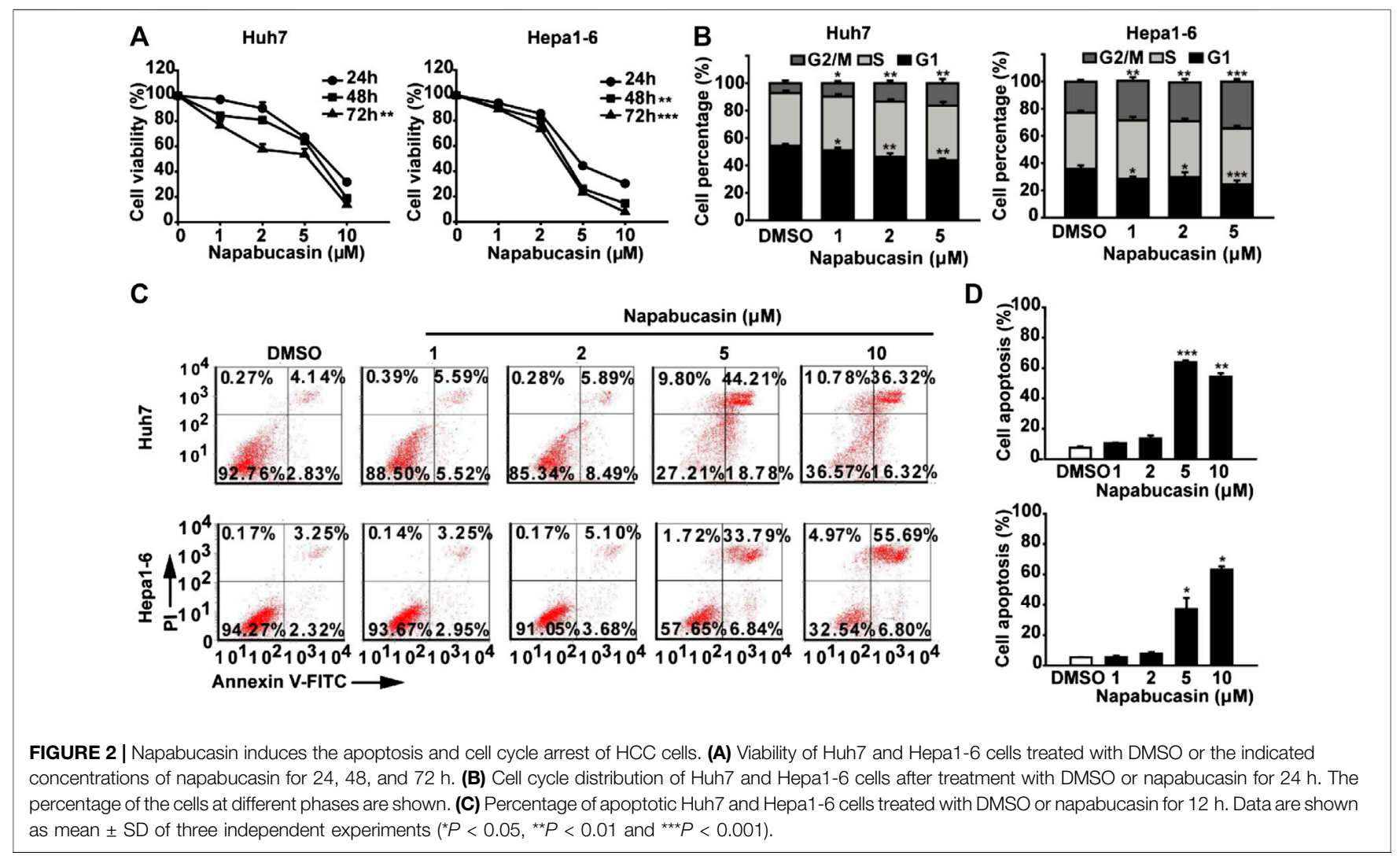



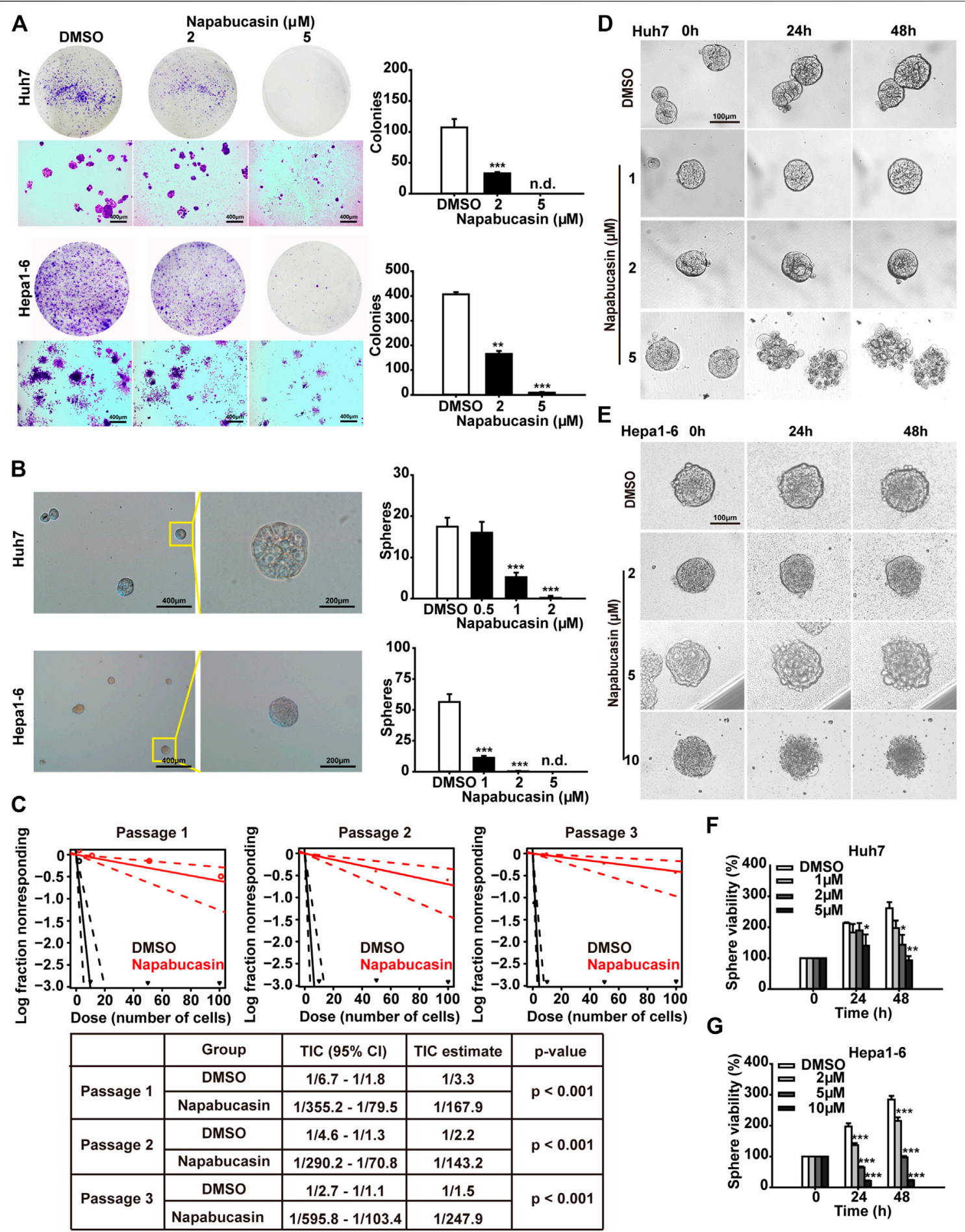

FIGURE 3 | Napabucasin suppresses sphere formation and viability of HCC cells. (A) Representative images of crystal violet-stained colonies (>100 cells) of Huh7 and Hepa1-6 cells after treatment with DMSO or indicated concentrations of napabucasin. (B) Representative images of multicellular spheroids larger than $80 \mu \mathrm{m}$ (left) and quantification of spheroids derived from DMSO- or napabucasin-treated Huh7 and Hepa1-6 cells (right). n.d., not detected. (C) Limiting dilution sphere assay of Huh7 cells under DMSO or $1 \mu \mathrm{M}$ napabucasin from passage one to three. ELDA analysis plot and relative table reporting the TIC percentage and estimation are shown. TIC: tumor-initiating cells. (D), (E). Representative real-time images of Huh7 and Hepa1-6 spheroids in the presence of DMSO or napabucasin. (F), (G). Viability of the DMSO or napabucasin-treated spheroids after 24 or $48 \mathrm{~h}$. Data are shown as mean $\pm \mathrm{SD}$ of three independent experiments $\left({ }^{\star} P<0.05\right.$, ${ }^{\star \star} P<0.01$ and $\left.{ }^{\star \star \star} P<0.001\right)$ 


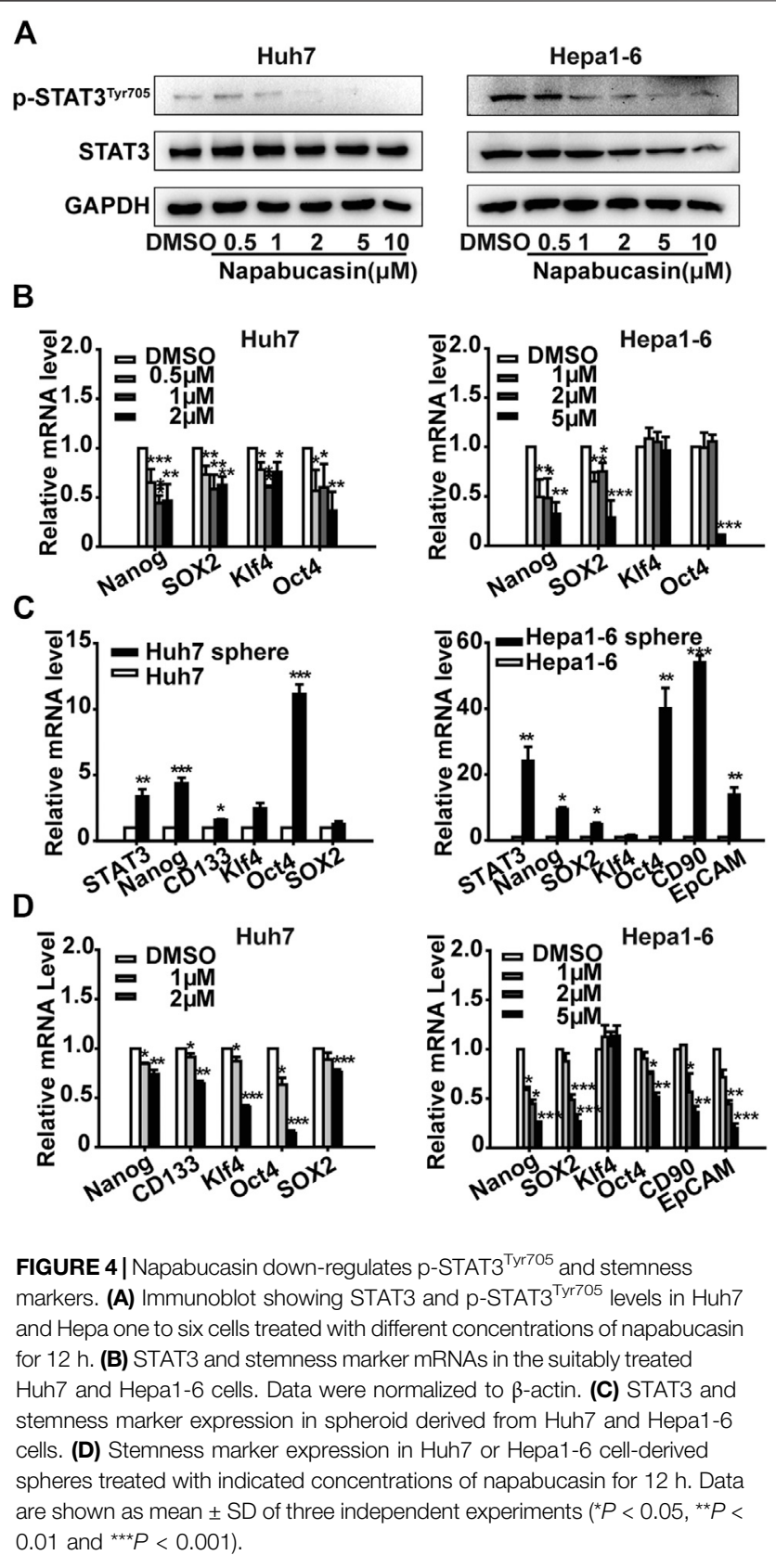

HCC cells in the presence of $8 \mu \mathrm{g} / \mathrm{ml}$ of polybrene (SigmaAldrich). Then HCC cells were collected for further analysis after culturing for $48 \mathrm{~h}$.

\subsection{Statistical Analysis}

Different groups were compared with Student's t-test or analysis of variance (ANOVA) as appropriate. The half maximal inhibitory concentration $\left(\mathrm{IC}_{50}\right)$ values were calculated by PASW Statistics 18.0.0. All data are expressed as mean \pm standard deviation (SD), and considered statistically significant at $p<0.05$.

\section{RESULTS}

\subsection{Napabucasin Inhibits HCC Cell Growth in vitro}

The potential cytotoxic effect of napabucasin on HCC cells was evaluated in terms of the proliferation ability of multiple human and murine HCC cell lines, and compared to that of oxaliplatin and the plant-derived STAT3 inhibitor cryptotanshinone (Liu et al., 2017; Qin et al., 2017; Ji et al., 2019; Zhu et al., 2019). As shown in Figure 1, all drugs significantly decreased the viability of HCC cells in a concentration-dependent manner. However, napabucasin exhibited a stronger suppressive effect compared to either cryptotanshinone or oxaliplatin, and had a significantly lower $\mathrm{IC}_{50}$ value (Table1). As shown in Figure 2A, napabucasin significantly decreased the viability of Huh7 and Hepa1-6 cells in a time- and concentration-dependent manner. Consistent with this, the proportion of napabucasin-treated cells in the G1-phase was significantly lower compared to that of the untreated controls, which corresponded to an increase in the G2/ M-phase cells $(P<0.05$; Figure 2B). Furthermore, the apoptosis rates in Huh7 cells increased to $11.11 \%, 14.38 \%$ and $62.99 \%$ respectively within $12 \mathrm{~h}$ of exposure to 1,2 and $5 \mu \mathrm{M}$ napabucasin (Figure 2C), and similar results were obtained with Hepa1-6 cells as well. Taken together, napabucasin inhibited HCC cell growth in a concentration-dependent manner by inducing apoptosis and G2/M-phase arrest.

\subsection{Napabucasin Decreases Colony and Spheroid Formation Abilities of HCC Cells}

To determine the potential effects of napabucasin on HCC metastasis, we assessed the colony-forming capacity of these cells following drug treatment. As shown in Figure 3A, napabuacsin significantly decreased the number and size of colonies formed by both Huh7 and Hepa1-6 cells in a concentration-dependent manner. Since metastasis is dependent on CSCs, we next analyzed the ability of the napabucasin-treated HCC cells to form spheroids in suspension. Compared to the DMSO-treated controls, napabucasin blocked spherogenesis of HCC cells (Figure 3B), and such ability is further enhanced along with the serial passages (Figure 3C), indicating that this drug can inhibit the self-renewal and proliferation of the CSC-like cells. Furthermore, the viability of the multicellular spheroids derived from Huh7 cells was significantly reduced by napabucasin, and the cell masses were obliterated at the high concentration of $5 \mu \mathrm{M}$ (Figures 3D,F). Similar results were seen with the Hepa1-6 cells (Figures 3E,G). Taken together, napabucasin showed an inhibitory effect on HCC metastasis, and the "stemness-high" HCC cells were particularly sensitive to the drug.

\subsection{Napabucasin Downregulates p-STAT3 ${ }^{\text {Tyr705 }}$ and Stemness Markers in HCC Cells}

The TCGA database showed an elevated expression of stemness makers such as Nanog, SOX2, Oct4 and CD90 in HCC patients 

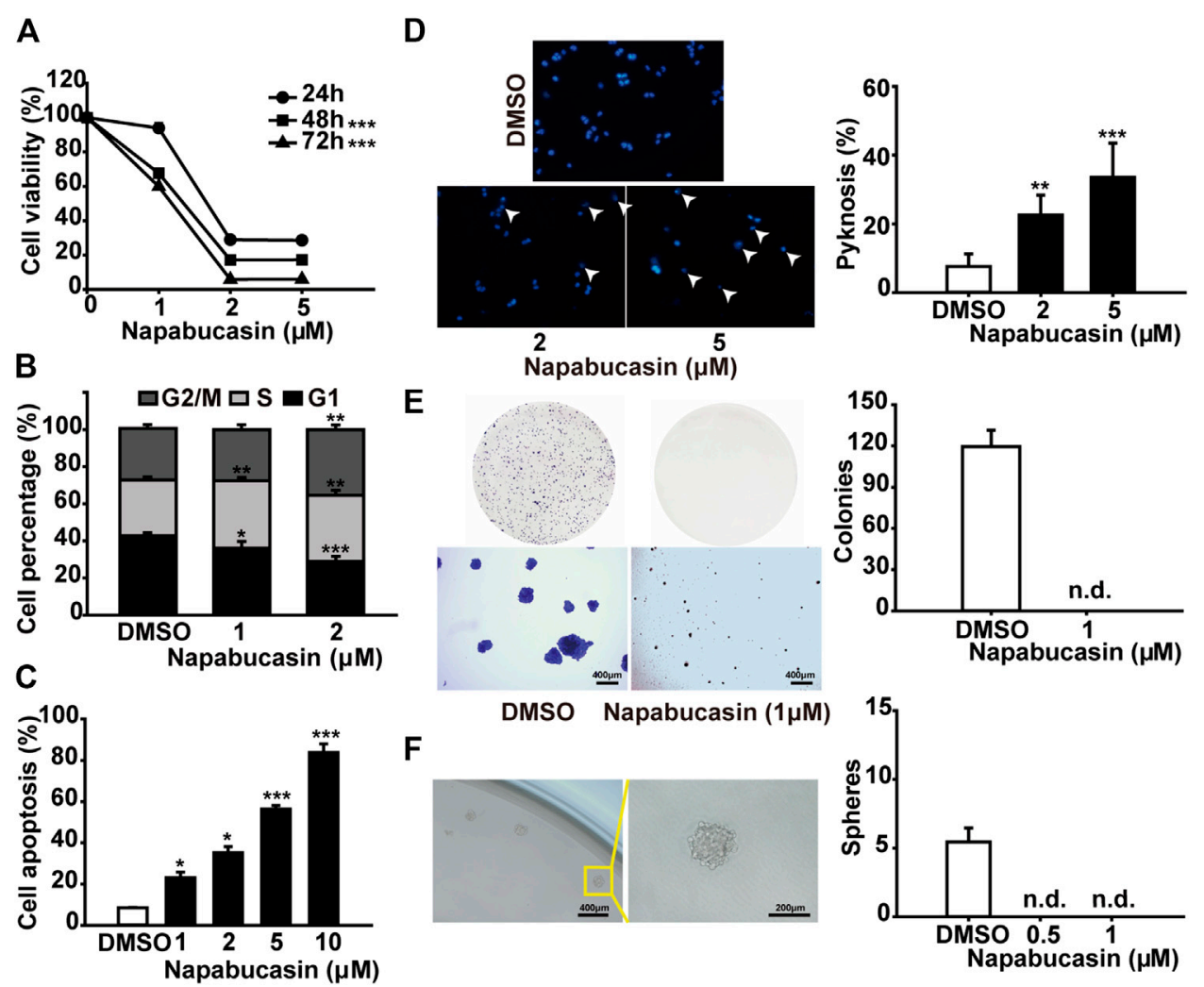

$\mathbf{F}$
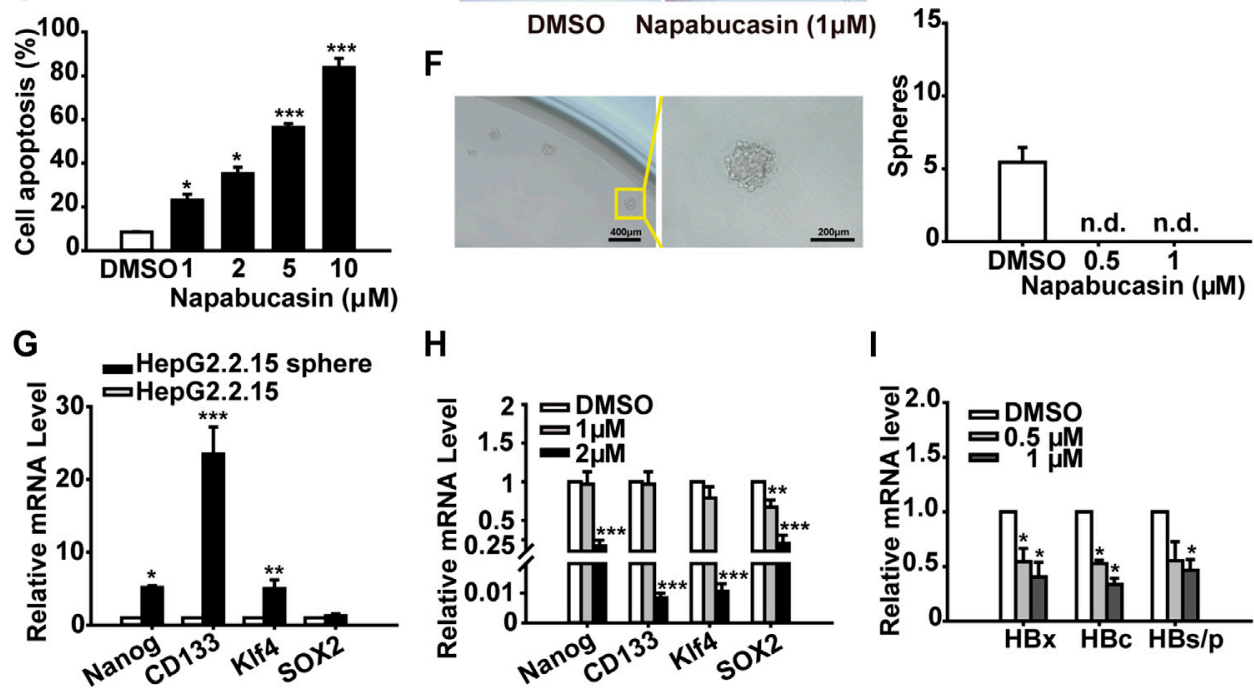

H

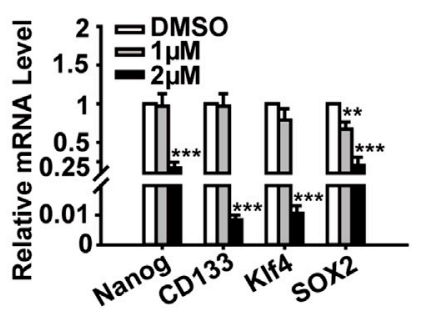

I

FIGURE 5 | Napabucasin induces apoptosis in HBV $\mathrm{HCC}^{+}$cells and decreases spheroid formation. (A) Viability of HepG2.215 cells treated with DMSO or indicated concentrations of napabucasin for 24, 48 and $72 \mathrm{~h}$. (B) Cell cycle distribution of HepG2.215 cells treated with DMSO or napabucasin for $24 \mathrm{~h}$. (C) Percentage of apoptotic HepG2.215 cells following treatment with DMSO or napabucasin for 12 h. (D) Representative images ( $\times 40$ magnification) of Hoechst 33342 -stained HepG2.215 cells treated with napabucasin for $6 \mathrm{~h}$ (left) and quantification of pyknosis (right). (E) Representative images of colonies formed by DMSO or napabucasin-treated HepG2.2.15 cells (left) and quantification of colonies (right). (F) Representative images of multicellular spheroids larger than $80 \mu \mathrm{m}$ (left) and quantification of spheroids derived from DMSO- or napabucasin-treated HepG2.215 cells (right). (G) Stemness marker expression in the spheres derived from HepG2.215 cells. (H) Stemness marker expression in HepG2.215 cell-derived spheres treated with indicated concentrations of napabucasin for $12 \mathrm{~h}$. Data were normalized to $\beta$-actin. (I) HBx, HBc and HBs/p levels in HepG2.215 cells treated with napabucasin for $12 \mathrm{~h}$. Data are shown as mean \pm SD of three independent experiments $\left({ }^{\star} P<0.05,{ }^{\star \star} P<0.01\right.$ and $\left.{ }^{\star \star *} P<0.001\right)$. n.d., not detected.

(Supplementary Figure 1). In vitro, the mRNA levels of Nanog, CD133, SOX2, Klf4, Oct4 and SOX2 were significantly decreased by STAT3 interference in HCC cells (Supplementary Figure 2). To further elucidate the molecular mechanisms underlying napabucasin action, we next analyzed the changes in the expression levels of STAT3 and stemness markers in the napabucasin-treated cells. Napabucasin decreased $p$-STAT3 $3^{\text {Tyr705 }}$ levels in Huh7 and Hepa1-6 cells in a concentration-dependent manner, but had little effect on total STAT3 expression (Figure 4A). In addition, the expression levels of Nanog, SOX2, Klf4 and Oct4
mRNA were also significantly decreased in the napabucasin-treated HCC cells in a concentration-dependent manner (Figure 4B). Furthermore, compared to the adherent cultures, STAT3, Nanog and Oct4 mRNAs were significantly upregulated in the Huh7 and Hepa1-6 cell-derived spheroids grown in suspension (Figure 4C), which was decreased by napabucasin in a concentration-dependent manner (Figure 4D). Therefore, napabucasin specifically inhibits the stemness-high HCC cells by inactivating STAT3 and downregulating multiple CSC-related transcription factors, and is expected to yield therapeutic effects in clinical. 


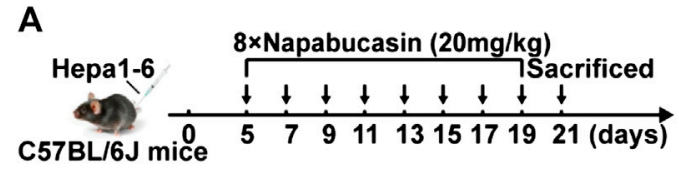

B

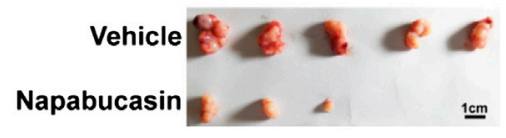

D

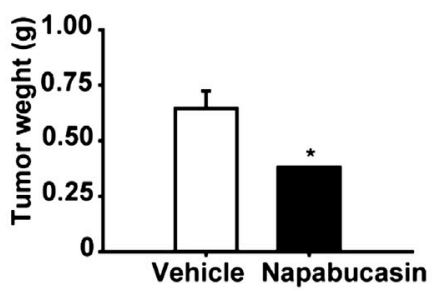

$\mathbf{F}$
C
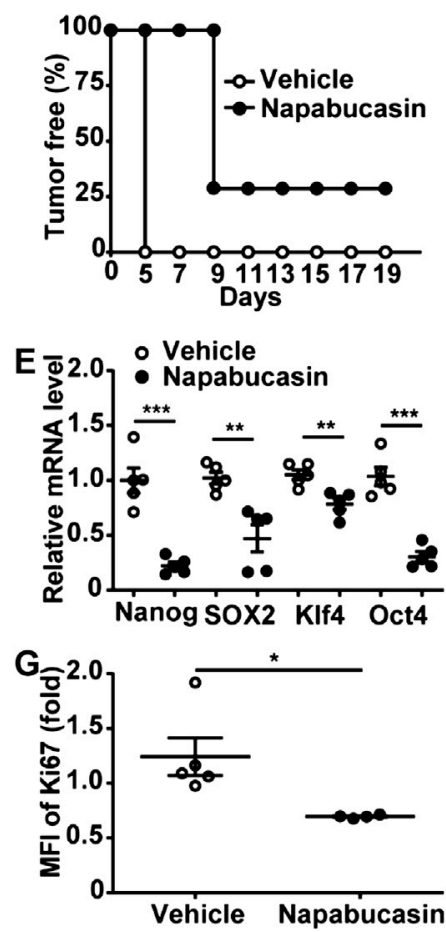

FIGURE 6 | Napabucasin inhibits HCC homograft growth in vivo. (A) C57BL/6J mice were inoculated with $5 \times 10^{6}$ Hepa1-6 cells in the left axilla, and intraperitoneally injected with napabucasin $(20 \mathrm{mg} / \mathrm{kg}$ ) or vehicle 5 days post-inoculation every 2 days for a total of eight injections. (B) Representative images of tumor tissues. (C) The percentage of tumor free mice. (D) Tumor weight of the suitably treated mice. (E) Stemness marker expression in the tumor cells. Data were normalized to $\beta$-actin. (F) Frequency of Ki67 ${ }^{+}$cells in the tumors. (G) Ki67 levels in tumor cells from mice treated as above. MFI, mean fluorescence index. Data represents the mean $\pm \mathrm{SD}$ of $\mathrm{n}=5$ and were analyzed with one-way ANOVA $\left({ }^{\star} P<0.05,{ }^{\star \star} P<0.01\right.$ and $\left.{ }^{\star \star \star} P<0.001\right)$.

\subsection{Napabucasin Induces $\mathrm{HBV}^{+} \mathrm{HCC}$ Cell Apoptosis and Decreases Spheroid Formation}

Hepatitis B virus (HBV) infection is the most common underlying cause of HCC worldwide, and the HBV regulatory protein X ( $\mathrm{HBx})$ acts as a positive regulator of STAT3 (Roca Suarez et al., 2018). Therefore, we also analyzed the anti-tumor effects of napabucasin on the $\mathrm{HBV}^{+} \mathrm{HepG} 2.2 .15$ cells. Consistent with the findings on Huh7 and Hepa1-6 cells, napabucasin significantly decreased the proliferation rates (Figure 5A), induced G2/M-phase arrest (Figure 5B), and increased apoptosis rates (Figures 5C,D) in the HepG2.2.15 cells in a time- and concentration-dependent manner. Notably, napabucasin could induce HepG2.2.15 cell apoptosis within $4 \mathrm{~h}$ (Supplementary Figure 3). Furthermore, napabucasin markedly reduced the colony forming capacity (Figure 5E) and blocked spherogenesis (Figure 5F) of HepG2.2.15 cells. The levels of Nanog, CD133, Klf4 and Sox-2 were increased in HepG2.2.15 spheroids (Figure 5G), which could be decreased by napabucasin treatment (Figure 5H). Interestingly, napabucasin also restricted HBV replication in the HepG2.2.15 cells by inhibiting $\mathrm{HBx}, \mathrm{HBV}$ core protein $(\mathrm{HBc})$, hepatitis $\mathrm{B}$ surface antigen (HBs) and HBV polymerase (HBp) (Figure 5I).
Taken together, napabucasin can be considered for the treatment of $\mathrm{HBV}^{+} \mathrm{HCC}$.

\subsection{Napabucasin Inhibits HCC Homografts in vivo}

To evaluate the anti-HCC effect of napabucasin in vivo, Hepa1-6 homografts were established in mice that were subsequently treated with $20 \mathrm{mg} / \mathrm{kg}$ napabucasin or placebo (Figure 6A). Napabucasin treatment significantly reduced tumor volumes compared to that in the placebo group (Figure 6B), and the homografts regressed completely in two of the seven mice (Figure 6C). Furthermore, the final tumor weights in the vehicle and napabucasin groups were $0.645 \pm 0.08 \mathrm{~g}$ and $0.38 \pm 0.01 \mathrm{~g}$ respectively (Figure 6D). In line with the in vitro findings, napabucasin down-regulated Nanog, SOX2, Klf4 and Oct4 in the tumor cells (Figure 6E), and also decreased the proportion of $\mathrm{Ki}^{+} 7^{+}$tumor cells (Figures 6F,G). Since most HCC cases are detected in the advanced stages, we also analyzed the effect of napabucasin on the Hepal-6 homografts 12 days post-inoculation (Figure 7A). As shown in Figures 7B and 7C, napabucasin did not significantly affect tumor growth compared to the placebo. However, napabucasin treatment increased the proportion of apoptotic tumor cells as well as the 

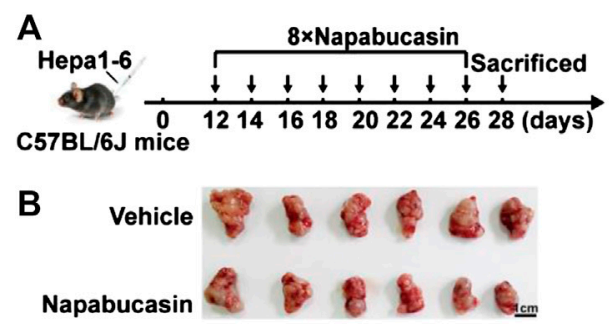

E

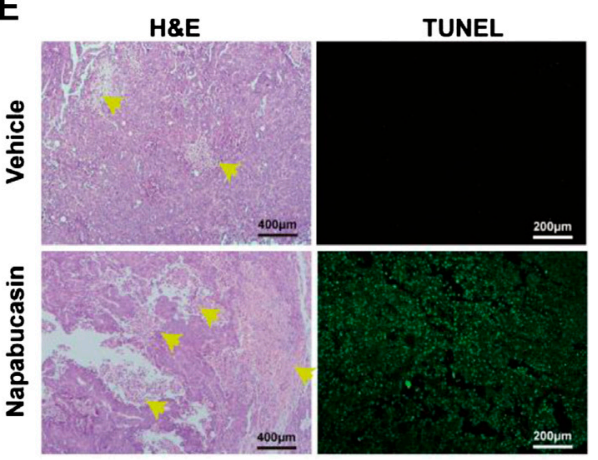

C

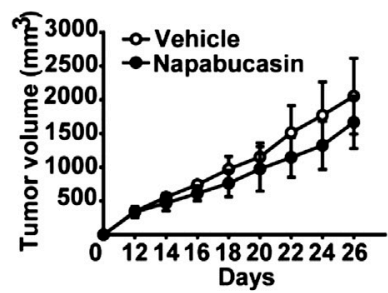

$F$

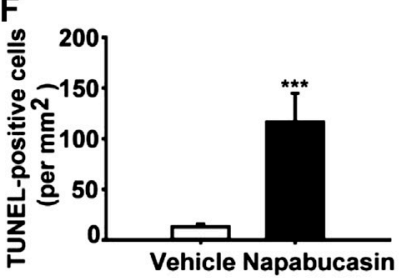

D

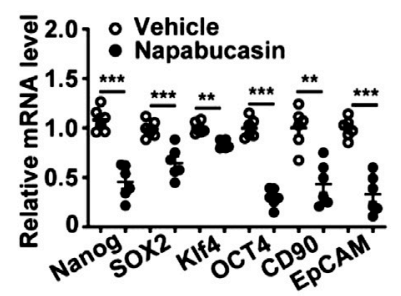

G

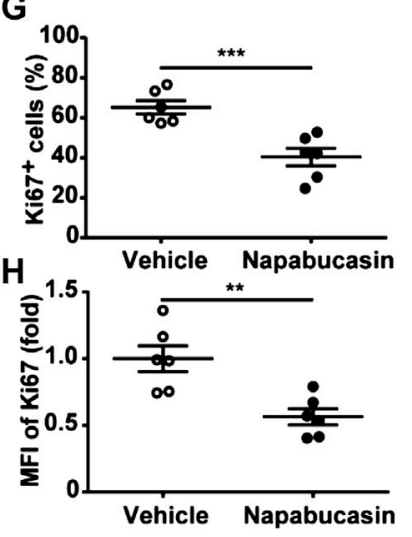

FIGURE 7 | Napabucasin induces necrosis in Hepa1-6 homografts in advanced stages. (A) C57BL/6J mice were inoculated with $5 \times 10^{6}$ Hepa1-6 cells in the left axilla, and intraperitoneally injected with napabucasin $(20 \mathrm{mg} / \mathrm{kg}$ ) or vehicle 12 days post-inoculation every 2 days for a total of eight injections. (B) Representative images of tumor tissues. (C) Tumor growth curves during the experimental period. (D) Stemness marker expression in tumor cells. Data were normalized to $\beta$-actin. (E) Representative images of H\&E and TUNEL-stained tumor tissues. (F) Quantification of TUNEL-positive cells per $\mathrm{mm}^{2}$ in tumor tissues from suitably treated mice.

(G) The frequency of Ki67 $7^{+}$cells in tumor tissues. (H) Ki67 levels in tumor cells. MFI, mean fluorescence index. Data represents the mean \pm SD of $n=6$ and were analyzed with ANOVA $\left({ }^{\star \star} P<0.01\right.$ and $\left.{ }^{\star \star \star} P<0.001\right)$.

necrotic area in the tumor tissues (Figure 7D). Furthermore, napabucasin down-regulated Nanog, SOX2, Klf4, Oct4, CD90 and EpCAM, and decreased the percentage of $\mathrm{Ki}^{+} 7^{+}$cells in the tumors (Figures 7E-G). Taken together, napabucasin was effective against both early and advanced stage HCC homografts in vivo.

\section{DISCUSSION}

Recent advances in surgical, systemic and locoregional therapies has improved the short-term survival of HCC patients. However, prognosis is still dismal due to high recurrence rates (Colagrande et al., 2016; Dimitroulis et al., 2017). CSCs are rare cells interspersed within the tumor bulk, and are responsible for chemo- and radio-resistance, and post-therapeutic relapse. In addition, long-term exposure to chemotherapeutic drugs or radiation often induces de-differentiation of mature cancer cells to stem-like phenotypes (Karakasiliotis and Mavromara, 2015; Chen et al., 2017a). Therefore, preventing CSCs formation is a viable strategy for preventing cancer relapse and metastasis.

The main cause of HCC-related mortality is the resistance to radiotherapies or chemotherapeutic drugs (Le Grazie et al., 2017). Furthermore, the advanced stage tumors are usually not resectable and therefore have limited treatment options. Sorafenib, currently the only targeted drug approved for systemic treatment of advanced HCC, confers a modest survival benefit (Dika and Abou-Alfa, 2017). Oxaliplatin is a platinum-based agent that blocks DNA replication and transcription, and has been approved for transcatheter arterial chemoembolization (TACE) along with epirubicin, oxaliplatin, mitomycin and lipiodol to treat HCC in China (Li et al., 2017). However, CSCs-induced resistance to oxaliplatin is a leading cause of combination treatment failure. Cryptotanshinone, a natural compound extracted from the root of Salvia miltiorrhiza Bunge, is a potential STAT3 inhibitor with antineoplastic effects (Chen et al., 2017b). It also induces apoptosis in the PGE2-treated HA22T HCC cells, and is therefore a novel drug for HCC treatment (Chang et al., 2018). Napabucasin effectively inhibits STAT3 and its downstream genes, including those regulating stemness ( $\mathrm{Li}$ et al., 2015). It has been shown to suppress spherogenesis of or kill stemness-high cells isolated from head and neck, colon and pancreatic cancer cell lines (MacDonagh et al., 2018; Beyreis et al., 2019; Han et al., 2019). Furthermore, napabucasin also prevented metastasis in a spontaneous liver metastasis model of colorectal cancer, and suppressed relapse in a pancreatic cancer xenograft model ( $\mathrm{Li}$ et al., 2015). We found napabucasin showed stronger cytotoxic effects on HCC cells compared to oxaliplatin and cryptotanshinone, and its $\mathrm{IC}_{50}$ was significantly lower.

CSCs drive cancer initiation, progression, metastasis, recurrence and drug resistance (Chen et al., 2013), and express Nanog, CD133, CD90, SOX2, EpCAM, CD44, Klf4 and Oct4, 


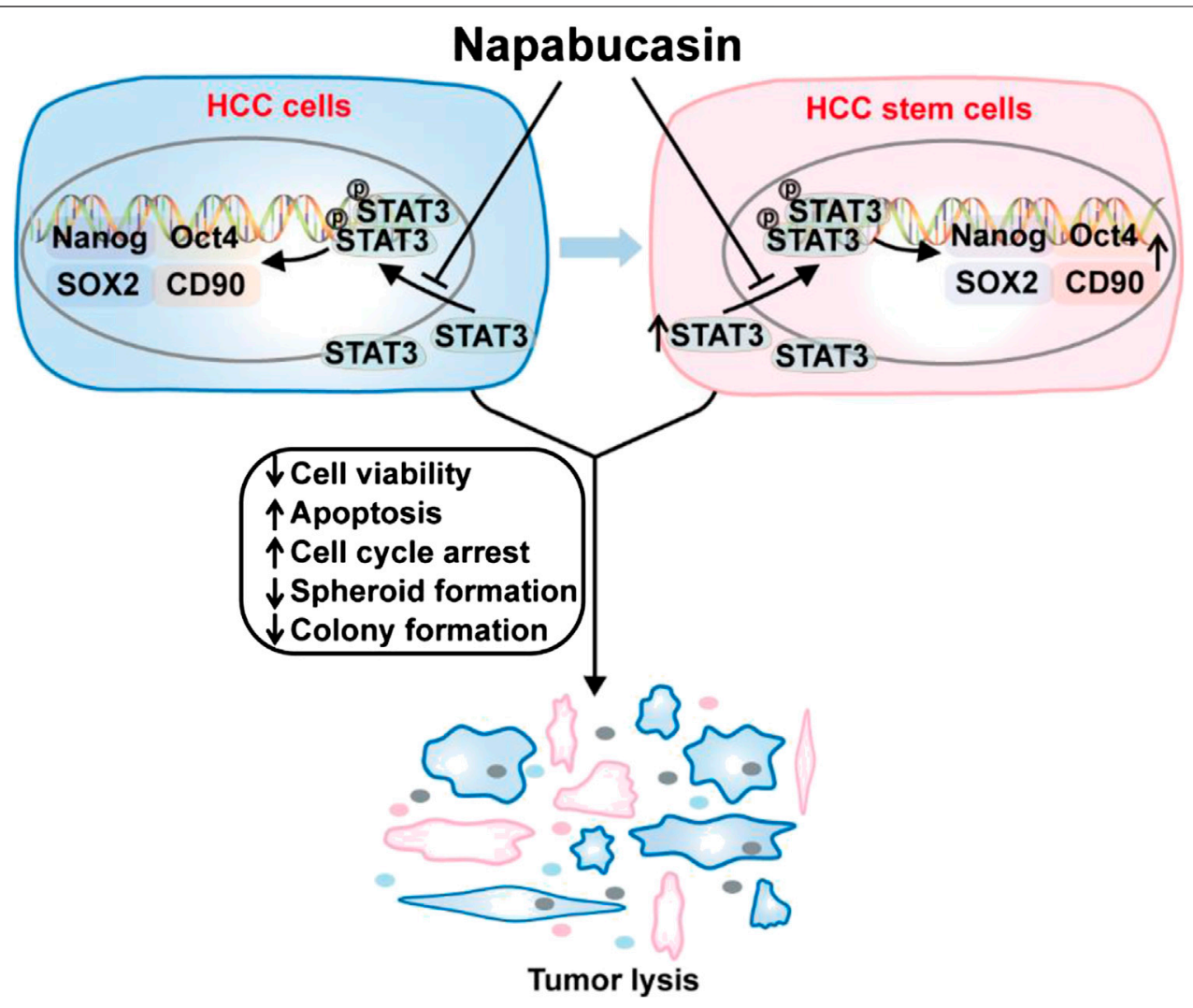

FIGURE 8 | Napabucasin reduces cancer stem cell characteristics in hepatocellular carcinoma. Napabucasin significantly decreased the viability of HCC cells in vitro by inducing apoptosis and cell cycle arrest. In addition, it suppressed CSC-related gene expression and spheroid formation in vitro, indicating depletion of CSCs. Further, napabucasin also restricted $\mathrm{HBV}$ replication in $\mathrm{HBV}^{+} \mathrm{HCC}$ cells.

some of which may functionally support liver CSC phenotypes like invasiveness and chemoresistance (Yamashita and Wang, 2013). For instance, EpCAM ${ }^{+}$HCC patients have lower overall and relapse free survival rates compared to the EpCAM ${ }^{-}$HCC patients (Sung et al., 2016; Zhou et al., 2016). In addition, high levels of CD133 and CD44 are associated with unfavorable prognosis of HCC (Zhao et al., 2016). $\mathrm{CD} 133^{+}$Huh7 or SMMC-7721 cells show higher proliferative and tumorigenic capacity compared to the $\mathrm{CD}_{133^{-}}$counterparts (Yin et al., 2007). Since the CSCs directly accelerate cancer relapse and metastasis, it is necessary to identify and target stemnessrelated molecules for effective anti-cancer therapy (Baccelli and Trumpp, 2012).

STAT3 is constitutively active in CSCs independent of the upstream signaling regulators. In addition, CSCs or stemnesshigh cancer cells are extremely sensitive to the direct inhibition of STAT3 but insensitive to the inhibition of upstream factors like Janus kinases (Li et al., 2015). Napabucasin diminished the CSClike traits of HCC cells, such as colony formation ability and spherogenesis, and killed the CSCs derived from Huh7 and Hepa1-6 spheres. Furthermore, napabucasin significantly downregulated the key stemness factors Nanog, SOX2, Oct4 and CD90 that are essential for maintaining pluripotency.
Thus, napabucasin can inhibit HCC growth by restraining the CSCs.

Hepatitis B and hepatitis $\mathrm{C}$ infection strongly predispose patients to chronic liver diseases and HCC, and are the major etiological factors of HCC in Asia and Africa (McGlynn and London, 2011; Mittal and El-Serag, 2013). Liver tumor biopsies from HBV-infected patients show EpCAM overexpression, and increased percentage of $\mathrm{Oct}^{+}$and Nanog${ }^{+}$cells (Arzumanyan et al., 2011). Consistent with this, ectopic expression of the HBx protein in HepG2 cells significantly upregulated Nanog, Klf4, Oct4, EpCAM and $\beta$-catenin (Arzumanyan et al., 2011). In a previous study, we found that blocking STAT3 signaling inhibited $\mathrm{HBV}^{+} \mathrm{HCC}$ cell growth in vitro by promoting apoptosis and cell cycle arrest. STAT3 silencing inhibited HBV replication, which decreased HBVdependent activation of STAT3 through a negative feedback loop, and augmented the anti-HCC effects (Yang et al., 2016). Napabucasin effectively suppressed the viability, clonogenic ability and stemness of the $\mathrm{HBV}^{+}$HepG2.2.15 cells, induced apoptosis and inhibited HBV replication, indicating the potential efficacy of napabucasin against $\mathrm{HBV}^{+} \mathrm{HCC}$.

The effects of napabucasin were also tested on early and advanced stage Hepa1-6 homografts in vivo. In the early stage model, napabucasin significantly inhibited tumor growth and 
decreased the percentage of proliferating cells, along with downregulating the stemness-related factors in the tumor tissues. However, napabucasin only marginally affected the growth of advanced tumor homografts despite inducing apoptosis and necrosis. It is possible that the effects of napabucasin in the advanced stages of HCC depend on its dose and bioavailability. Thus, dose-rate effects will have to be analyzed in order to improve the anti-tumor efficacy of napabucasin. Further, tumor tissues are composed of multiple cell types, including tumor cells, immune cells, endothelial cells, fibroblasts and so on, all of which are involved in the tumor progression (Fridman et al., 2012). For instance, a large number of regulatory $\mathrm{T}$ cells infiltrate in the tumor microenvironment, which is associated with the suppressive immune response to HCC cells (Togashi et al., 2019). Whether napabucasin can influence regulatory $\mathrm{T}$ cells or other cells in addition to HCC cells and then inhibit tumor growth by improving the tumor microenvironment need to be further explored.

In this study, we confirmed that napabucasin significantly decreased the viability of HCC cells in vitro by inducing apoptosis and cell cycle arrest. In addition, it suppressed CSC-related gene expression and spheroid formation in vitro, indicating depletion of CSCs. Interestingly, napabucasin also restricted HBV replication in $\mathrm{HBV}^{+} \mathrm{HCC}$ cells. The anti-neoplastic effects of napabucasin was also evident in homograft tumor-bearing mouse models (Figure 8). Napabucasin is promising as a drug or an adjuvant to standard chemo/immune-therapeutics against HCC.

\section{DATA AVAILABILITY STATEMENT}

The original contributions presented in the study are included in the article/Supplementary Material, further inquiries can be directed to the corresponding author.

\section{REFERENCES}

Arzumanyan, A., Friedman, T., Ng, I. O., Clayton, M. M., Lian, Z., and Feitelson, M. A. (2011). Does the hepatitis B antigen HBx promote the appearance of liver cancer stem cells? Canc. Res. 71 (10), 3701-3708. doi:10.1158/0008-5472.CAN-10-3951

Asrani, S. K., Devarbhavi, H., Eaton, J., and Kamath, P. S. (2019). Burden of liver diseases in the world. J. Hepatol. 70 (1), 151-171. doi:10.1016/j.jhep.2018.09.014

Baccelli, I., and Trumpp, A. (2012). The evolving concept of cancer and metastasis stem cells. J. Cell Biol. 198 (3), 281-293. doi:10.1083/jcb.201202014

Balogh, J., Victor, D., III, Asham, E. H., Burroughs, S. G., Boktour, M., Saharia, A., et al. (2016). Hepatocellular carcinoma: a review. J. Hepatocell. Carcinoma 3, 41-53. doi:10.2147/JHC.S61146

Bao, B., Ahmad, A., Azmi, A. S., Ali, S., and Sarkar, F. H. (2013). Overview of cancer stem cells (CSCs) and mechanisms of their regulation: implications for cancer therapy. Curr Protoc Pharmacol 61, 1-18. doi:10.1002/0471141755.ph1425s61

Beyreis, M., Gaisberger, M., Jakab, M., Neureiter, D., Helm, K., Ritter, M., et al. (2019). The cancer stem cell inhibitor napabucasin (BBI608) shows general cytotoxicity in biliary tract cancer cells and reduces cancer stem cell characteristics. Cancers 11 (3), 276. doi:10.3390/cancers11030276

Chang, J. H., Lin, C. H., Shibu, M. A., Chou, Y. C., Liu, J. Y., Chou, Y. H., et al. (2018). Cryptotanshinone (Dsh-003) from Salvia miltiorrhiza Bunge inhibits prostaglandin E2-induced survival and invasion effects in HA22T hepatocellular carcinoma cells. Environ. Toxicol. 33 (12), 1254-1260. doi:10. 1002/tox. 22633

\section{ETHICS STATEMENT}

The animal study was reviewed and approved by Ethical Committee of Shandong University.

\section{AUTHOR CONTRIBUTIONS}

YL: study conception, design and drafting of the manuscript. QH and HZ: lab work. QG: acquisition of data. JZ: study conception, design and critical revision.

\section{FUNDING}

The work was supported by grants from National Natural Science Foundation of China (No. 81972694, No. 81972686) and the National Major Science \& Technology Project for Control and Prevention of Major Infectious Diseases in China (No. 2018ZX10301401).

\section{ACKNOWLEDGMENTS}

We are grateful to the Pharmaceutical Biology Sharing Platform of Shandong University for the assistance and guidance in the flow cytometry assays.

\section{SUPPLEMENTARY MATERIAL}

The Supplementary Material for this article can be found online at: https:/www.frontiersin.org/articles/10.3389/fphar.2020.597520/ full\#supplementary-material

Chen, K., Huang, Y. H., and Chen, J. L. (2013). Understanding and targeting cancer stem cells: therapeutic implications and challenges. Acta Pharmacol. Sin. 34 (6), 732-740. doi:10.1038/aps.2013.27

Chen, X., Liao, R., Li, D., and Sun, J. (2017a). Induced cancer stem cells generated by radiochemotherapy and their therapeutic implications. Oncotarget 8 (10), 17301-17312. doi:10.18632/oncotarget.14230

Chen, Z., Zhu, R., Zheng, J., Chen, C., Huang, C., Ma, J., et al. (2017b). Cryptotanshinone inhibits proliferation yet induces apoptosis by suppressing STAT3 signals in renal cell carcinoma. Oncotarget 8 (30), 50023-50033. doi:10. 18632/oncotarget.18483

Colagrande, S., Inghilesi, A. L., Aburas, S., Taliani, G. G., Nardi, C., and Marra, F. (2016). Challenges of advanced hepatocellular carcinoma. World J. Gastroenterol. 22 (34), 7645-7659. doi:10.3748/wjg.v22.i34.7645

Daher, S., Massarwa, M., Benson, A. A., and Khoury, T. (2018). Current and future treatment of hepatocellular carcinoma: an updated comprehensive review. J Clin Transl Hepatol 6 (1), 69-78. doi:10.14218/JCTH.2017.00031

Dika, I. E., and Abou-Alfa, G. K. (2017). Treatment options after sorafenib failure in patients with hepatocellular carcinoma. Clin. Mol. Hepatol. 23 (4), 273-279. doi:10.3350/cmh.2017.0108

Dimitroulis, D., Damaskos, C., Valsami, S., Davakis, S., Garmpis, N., Spartalis, E., et al. (2017). From diagnosis to treatment of hepatocellular carcinoma: an epidemic problem for both developed and developing world. World J. Gastroenterol. 23 (29), 5282-5294. doi:10.3748/wjg.v23.i29.5282

Frank, N. Y., Schatton, T., and Frank, M. H. (2010). The therapeutic promise of the cancer stem cell concept. J. Clin. Invest. 120 (1), 41-50. doi:10.1172/JCI41004 
Fridman, W. H., Pages, F., Sautes-Fridman, C., and Galon, J. (2012). The immune contexture in human tumours: impact on clinical outcome. Nat. Rev. Canc. 12 (4), 298-306. doi:10.1038/nrc3245

Geschwind, J. H., and Chapiro, J. (2016). Sorafenib in combination with transarterial chemoembolization for the treatment of hepatocellular carcinoma. Gastroenterol. Hepatol. 12 (8), 504-506.

Han, D., Yu, T., Dong, N., Wang, B., Sun, F., and Jiang, D. (2019). Napabucasin, a novel STAT3 inhibitor suppresses proliferation, invasion and stemness of glioblastoma cells. J. Exp. Clin. Canc. Res. 38 (1), 289. doi:10.1186/s13046019-1289-6

Hu, Y., and Smyth, G. K. (2009). ELDA: extreme limiting dilution analysis for comparing depleted and enriched populations in stem cell and other assays. J. Immunol. Methods 347 (1-2), 70-78. doi:10.1016/j.jim.2009.06.008

Ji, Y., Liu, Y., Xue, N., Du, T., Wang, L., Huang, R., et al. (2019). Cryptotanshinone inhibits esophageal squamous-cell carcinoma in vitro and in vivo through the suppression of STAT3 activation. OncoTargets Ther. 12, 883-896. doi:10.2147/ OTT.S187777

Jiang, W., Peng, J., Zhang, Y., Cho, W. C., and Jin, K. (2012). The implications of cancer stem cells for cancer therapy. Int. J. Mol. Sci. 13 (12), 16636-16657. doi:10.3390/ijms131216636

Kamran, M. Z., Patil, P., and Gude, R. P. (2013). Role of STAT3 in cancer metastasis and translational advances. BioMed Res. Int. 2013, 421821. doi:10.1155/2013/421821

Karakasiliotis, I., and Mavromara, P. (2015). Hepatocellular carcinoma: from hepatocyte to liver cancer stem cell. Front. Physiol. 6, 154. doi:10.3389/ fphys.2015.00154

Karras, J. G., Wang, Z., Huo, L., Howard, R. G., Frank, D. A., and Rothstein, T. L. (1997). Signal transducer and activator of transcription-3 (STAT3) is constitutively activated in normal, self-renewing B-1 cells but only inducibly expressed in conventional B lymphocytes. J. Exp. Med. 185 (6), 1035-1042. doi:10.1084/jem.185.6.1035

Kim, J. W., Ye, Q., Forgues, M., Chen, Y., Budhu, A., Sime, J., et al. (2004). Cancerassociated molecular signature in the tissue samples of patients with cirrhosis. Hepatology 39 (2), 518-527. doi:10.1002/hep.20053

Lai, S. C., Chang, T. S., Su, Y. T., Wu, Y. C., Kuo, Y. C., Lan, P. C., et al. (2018). IL-6 regulates the OCT4 expression by epigenetic modulation in hepatocellular carcinoma. Canc. Res. 78 (13), 5223. doi:10.1158/1538-7445.AM2018-5223

Le Grazie, M., Biagini, M. R., Tarocchi, M., Polvani, S., and Galli, A. (2017). Chemotherapy for hepatocellular carcinoma: the present and the future. World J. Hepatol. 9 (21), 907-920. doi:10.4254/wjh.v9.i21.907

Li, D., Zhang, B., and $\mathrm{Hu}$, C. (2017). Oxaliplatin inhibits proliferation and migration of human hepatocellular carcinoma cells via GAS7C and the N-WASP/FAK/F-actin pathway. Acta Biochim. Biophys. Sin. 49 (7), 581-587. doi:10.1093/abbs/gmx046

Li, Y., Rogoff, H. A., Keates, S., Gao, Y., Murikipudi, S., Mikule, K., et al. (2015). Suppression of cancer relapse and metastasis by inhibiting cancer stemness. Proc. Natl. Acad. Sci. USA 112 (6), 1839-1844. doi:10.1073/pnas.1424171112

Liu, X., Xu, J., Wang, S., Yu, X., Kou, B., Chai, M., et al. (2017). Synergistic inhibitory effects on hepatocellular carcinoma with recombinant human adenovirus Aspp2 and oxaliplatin via p53-independent pathway in vitro and in vivo. Int. J. Oncol. 51 (4), 1291-1299. doi:10.3892/ijo.2017.4105

MacDonagh, L., Gray, S. G., Breen, E., Cuffe, S., Finn, S. P., O’Byrne, K. J., et al. (2018). BBI608 inhibits cancer stemness and reverses cisplatin resistance in NSCLC. Canc. Lett. 428, 117-126. doi:10.1016/j.canlet.2018.04.008

McGlynn, K. A., and London, W. T. (2011). The global epidemiology of hepatocellular carcinoma: present and future. Clin. Liver Dis. 15 (2), 223-243. doi:10.1016/j.cld.2011.03.006

Mittal, S., and El-Serag, H. B. (2013). Epidemiology of hepatocellular carcinoma: consider the population. J. Clin. Gastroenterol. 47 (l), S2-S6. doi:10.1097/MCG. ob013e3182872f 29

Phi, L. T. H., Sari, I. N., Yang, Y. G., Lee, S. H., Jun, N., Kim, K. S., et al. (2018). Cancer stem cells (CSCs) in drug resistance and their therapeutic implications in cancer treatment. Stem Cell. Int. 2018, 5416923. doi:10.1155/2018/5416923

Qin, S., Zhang, X., Guo, W., Feng, J., Zhang, T., Men, L., et al. (2017). Prognostic nomogram for advanced hepatocellular carcinoma treated with FOLFOX 4.
Asian Pac. J. Cancer Prev. 18 (5), 1225-1232. doi:10.22034/APJCP.2017.18.5. 1225

Rawla, P., Sunkara, T., Muralidharan, P., and Raj, J. P. (2018). Update in global trends and aetiology of hepatocellular carcinoma. Contemp. Oncol. 22 (3), 141-150. doi:10.5114/wo.2018.78941

Roca Suarez, A. A., Van Renne, N., Baumert, T. F., and Lupberger, J. (2018). Viral manipulation of STAT3: evade, exploit, and injure. PLoS Pathog. 14 (3), e1006839. doi:10.1371/journal.ppat.1006839

Samonakis, D. N., and Kouroumalis, E. A. (2017). Systemic treatment for hepatocellular carcinoma: still unmet expectations. World J. Hepatol. 9 (2), 80-90. doi:10.4254/wjh.v9.i2.80

Sonbol, M. B., Ahn, D. H., Goldstein, D., Okusaka, T., Tabernero, J., Macarulla, T., et al. (2019). CanStem111P trial: a Phase III study of napabucasin plus nabpaclitaxel with gemcitabine. Future Oncol. 15 (12), 1295-1302. doi:10.2217/fon2018-0903

Sun, X., Zhang, J., Wang, L., and Tian, Z. (2008). Growth inhibition of human hepatocellular carcinoma cells by blocking STAT3 activation with decoy-ODN. Canc. Lett. 262 (2), 201-213. doi:10.1016/j.canlet.2007.12.009

Sung, J. J., Noh, S. J., Bae, J. S., Park, H. S., Jang, K. Y., Chung, M. J., et al. (2016). Immunohistochemical expression and clinical significance of suggested stem cell markers in hepatocellular carcinoma. J Pathol Transl Med 50 (1), 52-57. doi:10.4132/jptm.2015.10.09

Togashi, Y., Shitara, K., and Nishikawa, H. (2019). Regulatory T cells in cancer immunosuppression - implications for anticancer therapy. Nat. Rev. Clin. Oncol. 16 (6), 356-371. doi:10.1038/s41571-019-0175-7

Won, C., Kim, B. H., Yi, E. H., Choi, K. J., Kim, E. K., Jeong, J. M., et al. (2015). Signal transducer and activator of transcription 3-mediated CD133 upregulation contributes to promotion of hepatocellular carcinoma. Hepatology 62 (4), 1160-1173. doi:10.1002/hep. 27968

Yamashita, T., and Wang, X. W. (2013). Cancer stem cells in the development of liver cancer. J. Clin. Invest. 123 (5), 1911-1918. doi:10.1172/JCI66024

Yang, Y., Zheng, B., Han, Q., Zhang, C., Tian, Z., and Zhang, J. (2016). Targeting blockage of STAT3 inhibits hepatitis B virus-related hepatocellular carcinoma. Canc. Biol. Ther. 17 (4), 449-456. doi:10.1080/15384047.2016.1156257

Yin, S., Li, J., Hu, C., Chen, X., Yao, M., Yan, M., et al. (2007). CD133 positive hepatocellular carcinoma cells possess high capacity for tumorigenicity. Int. J. Canc. 120 (7), 1444-1450. doi:10.1002/ijc.22476

Yin, X., Zhang, B. H., Zheng, S. S., Gao, D. M., Qiu, S. J., Wu, W. Z., et al. (2015). Coexpression of gene Oct4 and Nanog initiates stem cell characteristics in hepatocellular carcinoma and promotes epithelial-mesenchymal transition through activation of Stat3/Snail signaling. J. Hematol. Oncol. 8, 23. doi:10. 1186/s13045-015-0119-3

Zhao, Q., Zhou, H., Liu, Q., Cao, Y., Wang, G., Hu, A., et al. (2016). Prognostic value of the expression of cancer stem cell-related markers CD133 and CD44 in hepatocellular carcinoma: from patients to patient-derived tumor xenograft models. Oncotarget 7 (30), 47431-47443. doi:10.18632/oncotarget.10164

Zhou, Y., Wang, B., Wu, J., Zhang, C., Zhou, Y., Yang, X., et al. (2016). Association of preoperative EpCAM Circulating Tumor Cells and peripheral Treg cell levels with early recurrence of hepatocellular carcinoma following radical hepatic resection. BMC Canc. 16, 506. doi:10.1186/s12885-016-2526-4

Zhu, R., Wang, W., and Yang, S. (2019). Cryptotanshinone inhibits hypoxia/ reoxygenation-induced oxidative stress and apoptosis in renal tubular epithelial cells. J. Cell. Biochem. doi:10.1002/jcb.28609

Conflict of Interest: The authors declare that the research was conducted in the absence of any commercial or financial relationships that could be construed as a potential conflict of interest.

Copyright (C) $2020 \mathrm{Li}, \mathrm{Han}$, Zhao, Guo and Zhang. This is an open-access article distributed under the terms of the Creative Commons Attribution License (CC BY). The use, distribution or reproduction in other forums is permitted, provided the original author(s) and the copyright owner(s) are credited and that the original publication in this journal is cited, in accordance with accepted academic practice. No use, distribution or reproduction is permitted which does not comply with these terms. 